\title{
Quasi-periodic ELF/VLF wave emissions in the Earth's magnetosphere: comparison of satellite observations and modeling
}

\author{
D. L. Pasmanik ${ }^{1}$, E. E. Titova ${ }^{2}$, A. G. Demekhov ${ }^{1}$, V. Y. Trakhtengerts ${ }^{1}$, O. Santolik ${ }^{3}$, F. Jiricek ${ }^{4}$, K. Kudela ${ }^{5}$, and \\ M. Parrot ${ }^{6}$ \\ ${ }^{1}$ Institute of Applied Physics RAS, Nizhny Novgorod, Russia \\ ${ }^{2}$ Polar Geophysical Institute, Apatity, Russia \\ ${ }^{3}$ Charles University, Prague, Czech Republic and Institute of Atmospheric Physics, Prague, Czech Republic \\ ${ }^{4}$ Institute of Atmospheric Physics, Prague, Czech Republic \\ ${ }^{5}$ Institute of Experimental Physics, Slovak Acad. Sci., Kosice, Slovakia \\ ${ }^{6}$ CNRS/LPCE 45071 Orléans cedex 02, France
}

Received: 12 November 2003 - Revised: 9 September 2004 - Accepted: 15 September 2004 - Published: 22 December 2004

\begin{abstract}
We present the results of a case study of quasiperiodic (QP) ELF/VLF hiss emissions detected on board the Freja and Magion 5 satellites. Detailed analysis of available QP events revealed certain specific features of their dynamic spectra, which have not been reported earlier. In particular, we found an event with an increase in the frequency drift rate during the generation of a single element of QP emission, and an event with alteration of QP elements having different frequency drift rates. Another event demonstrates the possible relationship between QP hiss emissions and discrete VLF emissions. Properties of QP events are compared with parameters of energetic electrons and cold plasma, and other available data.

Possible scenarios for the formation of these emissions are discussed on the basis of self-consistent simulations of the cyclotron instability, employing the information obtained experimentally. It is shown that the generation regime of selfsustained pulsations can explain consistently our data set. We show that our numerical model is capable of explaining the mentioned specific features of the dynamic spectrum of QP emissions. Comparison of the modeling results with experimental data yields an estimate for the parameters not measured directly.
\end{abstract}

Key words. Magnetospheric physics (plasma waves and instabilities; energetic particles, precipitating; energetic particles, trapped)

\section{Introduction}

Quasi-periodic (QP) whistler emissions are wide-band emissions that are observed inside or near the plasmapause (see,

Correspondence to: D. L. Pasmanik

(pdl@aurora.appl.sci-nnov.ru) e.g. Helliwell, 1965; Sato et al., 1974; Hovner et al., 1977; Tixier and Cornilleau-Wehrlin, 1986; Hayakawa and Sazhin, 1992; Sazhin and Hayakawa, 1994; Smith et al., 1998). They are characterized by a periodic modulation of the wave intensity with typical periods from several seconds up to a few minutes. Generation of QP emissions is usually accompanied by the precipitation of energetic particles, which are also modulated by the same period.

Sato et al. (1974) divided QP emissions into two classes: QP1 emissions which are closely associated with geomagnetic pulsations of the same period and QP2 emissions which do not correlate with geomagnetic pulsations. Sato and Fukunishi (1981) categorized QP emissions on the basis of their spectral forms. They divided QP emissions into five types based on frequency-time (f-t) spectra and suggested a phenomenological model for explanation of different types of the $\mathrm{f}$-t spectrum of QP emissions.

In this paper we restrict our consideration to the QP2 class emissions. A typical spectrum of QP2 emissions has the shape of periodic bursts of the wave intensity with constant positive frequency drift during the generation of a single QP element. A gradual increase or decrease in the frequency drift from one element to another may occur during an event. It is also known that the generation of QP emissions can be accompanied by the generation of discrete emissions.

Bespalov and Trakhtengerts (1976) and Davidson (1979) proposed an idea to explain the QP modulation of the wave intensity and electron precipitation in the absence of any external periodic disturbances by the periodic wave generation in the regime of relaxation oscillations. Such regimes may take place in the presence of a constant source of energetic electrons with transverse anisotropic distribution function. Further studies showed (Bespalov, 1981) that under certain conditions, the relaxation oscillations can turn into self-sustaining oscillations. The results obtained on the basis 


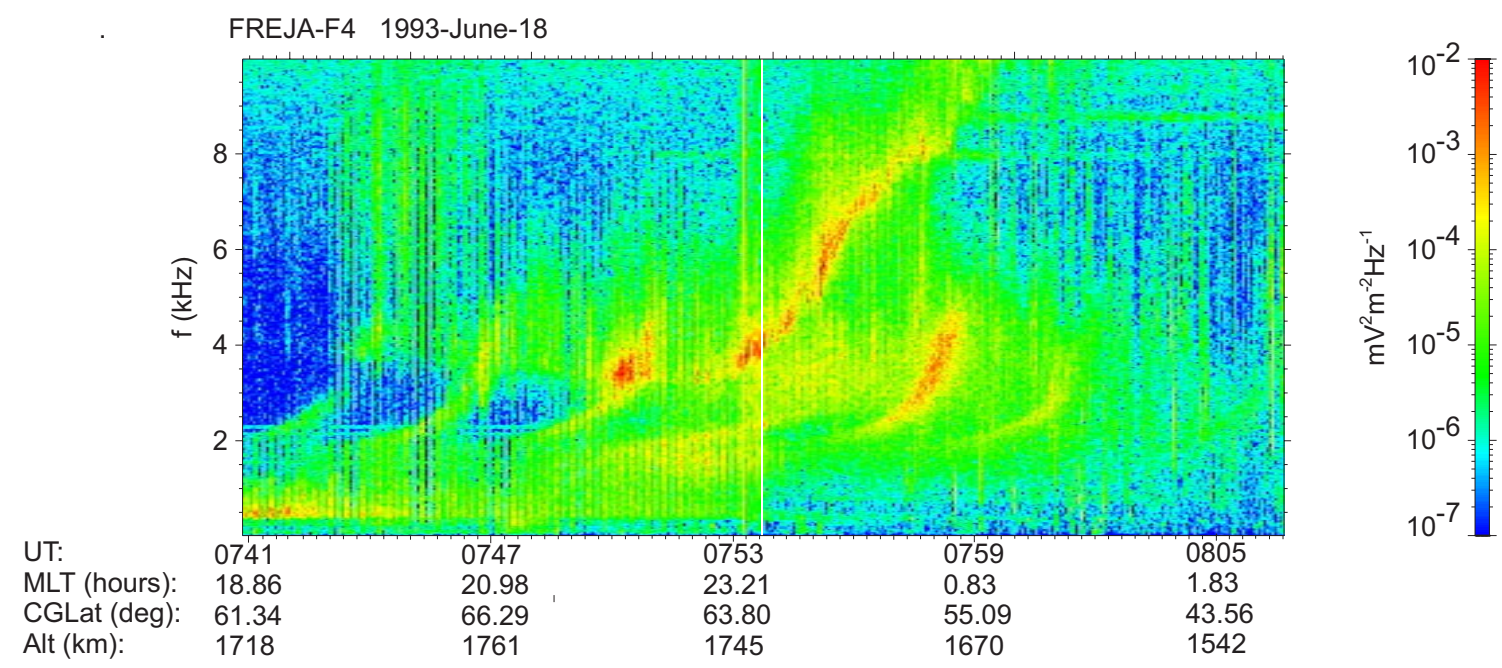

Fig. 1. Frequency-time spectra of the electric component of VLF emissions observed on board Freja satellite on 18 June 1993 . Peculiarity of the dynamic spectrum in this case is an increase in the frequency drift rate during generation of a single element of QP emission.

of this model allow one to explain the general properties and characteristic time scales of QP2 emissions, but in this model an approximation of constant shapes (not amplitudes) of the wave spectrum and energetic particle distribution were used. Similar results were obtained by Davidson (1986) on the basis of a phenomenological model, which also neglects the wave spectrum dynamics.

Trakhtengerts et al. (1986) and Demekhov and Trakhtengerts (1994) developed more rigorous kinetic model of flow cyclotron maser (FCM) to explain pulsating aurorae patches. This model describes different periodic regimes of wave generation with different shapes of the dynamic spectrum and can also be used for modeling the generation of QP emissions. Further study of this model was performed by Pasmanik et al. (2004), where the dependence of possible generation regimes on the parameters of the energeticelectron source are analyzed. The results of Pasmanik et al. (2004) demonstrate that the FCM model can produce a variety of periodic regimes of ELF/VLF generation with different types of dynamical spectra. These results enable one to relate numeric and functional parameters of the model with features of the generation regimes.

In this paper, we analyze some particular events of QP emissions, detected on board the Freja and Magion 5 satellites, which demonstrate certain specific features. Using the general results obtained by Pasmanik et al. (2004), we choose suitable parameters of the simulation model, such as source characteristics and cold plasma density, for each experimental case. Good correspondence between the observations and simulations is demonstrated.

\section{Observations}

Several samples of experimental data for QP emissions detected on board the low-apogee Freja satellite and highapogee Magion 5 satellite are analyzed in this section. We pay particular attention to the dynamic spectrum shapes of the observed emissions and try to interpret them below using self-consistent modeling. In some respect, the data of Freja and Magion 5 complement each other. Namely, Freja events are more numerous and show a larger variety of specific features of the dynamic spectra; some of these features are new and, to our knowledge, have not been reported earlier. On the other hand, the low-altitude location of the Freja orbit makes it impossible to localize the magnetospheric source of the observed QP emissions and, thus, to obtain plasma parameters in the source. The high-apogee Magion 5 satellite detected QP emissions fairly close to the geomagnetic equator and, therefore, it was possible to assume that the measured plasma parameters were close to those in the source.

\subsection{QP events observed by Freja}

The Freja satellite operated between October 1992 and October 1996 on a low-altitude orbit with an apogee of $1756 \mathrm{~km}$ and an inclination of $63^{\circ}$. In this study, we use the measurements of electric and magnetic fields in two frequency bands (up to $2 \mathrm{kHz}$ and up to $16 \mathrm{kHz}$ ) from the wave experiment $\mathrm{F} 4$ (Holback et al., 1994).

We have selected 3 orbits when QP emissions were detected on board Freja satellite. Dynamic spectra of QP emissions on all 3 orbits show rising tones, the magnetic activity was very low (Kp index did not exceed $1+$, and was $\mathrm{Kp}=0-0+$ for these QP events). It must be said that Freja also observed $\mathrm{QP}$ emissions at much higher magnetic activity. According to the categorization suggested by Sato and Fukunishi (1981), $\mathrm{QP}$ emissions with rising-tone elements detected during low magnetic activity belong to the QP2 class emissions, which are usually not correlated to geomagnetic pulsations on the ground. QP2 emissions occur in a wide local time interval from morning to midnight (Sato and Fukunishi, 1981), as it is true for these three Freja events. Particular attention in 


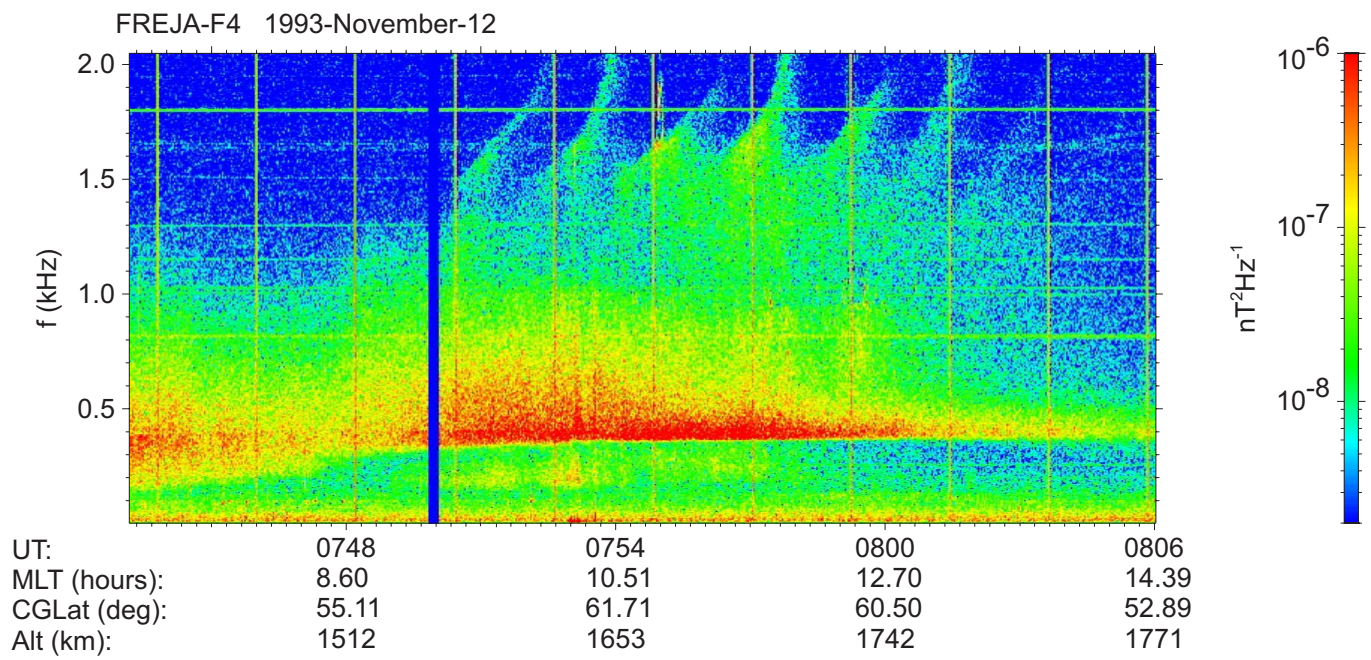

Fig. 2. Frequency-time spectra of the magnetic component of VLF emissions observed on board Freja satellite on 12 November 1993. Peculiarity of the dynamic spectrum in this case is the alteration of QP elements with different frequency drift rates.

our studies for the Freja satellite is paid to the events with some interesting peculiarities of the observed dynamic spectra. These events are discussed below.

Event of 18 June 1993: Figure 1 shows the power spectrogram of the electric component of VLF emissions detected by the Freja satellite on 18 June 1993, between 07:42 UT and 08:06 UT. Freja passed the evening-night sector at an altitude of $1700 \mathrm{~km}$, from subauroral to auroral latitudes, then it returned to subauroral latitudes. An intense LHR hiss was detected by Freja from 07:52 UT to 08:02 UT in the frequency range $3-8 \mathrm{kHz}$. A fast increase in the LHR-hiss frequency was related to the spacecraft motion from the ionospheric trough region to the plasmasphere.

As is seen from Fig. 1, the frequency range of QP emissions is $2-5 \mathrm{kHz}$, and their period is about $200 \mathrm{~s}$. It is also seen that the QP emissions were observed in a wide range of corrected geomagnetic latitudes (55-66 ${ }^{\circ}$ CGLat) and magnetic local times (19:00-24:00 MLT). The spectrum of the detected QP emissions preserved the same features during the entire pass, which implies that the observation zone was formed due to propagation of the emissions from a magnetospheric source, similarly as it takes place for chorus emissions (Jiricek et al., 1981). Note that the center of the QP observation zone, where they reach the maximum amplitude, is located near $60^{\circ}$ in the region of the ionospheric trough (indicated by the minimum of the LHR frequency), i.e. near the plasmapause projection.

A specific feature of the dynamic spectrum in this case is an increase in the frequency drift rate during the generation of a single element of QP emission.

Event of 12 November 1993: Figure 2 shows frequencytime spectra of the magnetic component of VLF emissions observed by Freja on 12 November 1993 . The spacecraft moved from low to auroral latitudes, reached the maximum CGlat of $61^{\circ}$ about 07:54 UT, and moved back to equatorial latitudes. QP emissions were detected at $57-61^{\circ}$ CGlat, in a wide range of MLT from 09:00 to 13:00. The geomagnetic disturbance was low, $\mathrm{Kp}=1+$. The frequency range of $\mathrm{QP}$ emissions is $1.3-2 \mathrm{kHz}$, and their period is about $100 \mathrm{~s}$.

In this case the specific feature of the dynamic spectrum is the alteration of QP elements with different frequency drift rates. Figure 2 clearly shows this feature, which can also be described as a superposition of a QP series with a smaller drift rate exactly in the middle of the other series with the same period but larger drift rate. Note that a variation in the frequency drift rate in QP emissions was observed by Sato and Fukunishi (1981), but that was a monotonic variation, i.e. either an increase or a decrease of the drift rate for each subsequent element for the entire QP event. Therefore, this study presents the first observation of a new type of QP dynamic spectrum.

Event 3 April 1995: Figure 3 shows the power spectrogram of the magnetic component of VLF emissions observed by Freja satellite on 3 April 1995, between 17:48 and 18:58 UT. QP emissions were detected in the morning sector (MLT $=08: 00-10: 00)$ at CGlat $=60-70^{\circ}$, during an extremely quiet period $(\mathrm{Kp}=0$ during the event and $\mathrm{Kp}<0+$ during the entire day of 3 April 1995). QP emissions were detected in the frequency range $1.3-1.6 \mathrm{kHz}$ with a period of $70-80 \mathrm{~s}$.

The specific feature of this event is that the majority of the QP elements are accompanied by additional short bursts (of several seconds in duration) at higher frequencies. These additional emission bursts are not noise; they have fine spectral structure, and sometimes one observes discrete emissions with rising tones. Figure $3 b$ presents a more detailed view of the spectrum of a QP element near 100 s (Fig. 3a). First, the emission spectrum is noise-like, and about 00:01:48, the power at higher frequencies becomes increased, and discrete elements appear. This observed relation of QP and discrete emissions will be discussed below. 


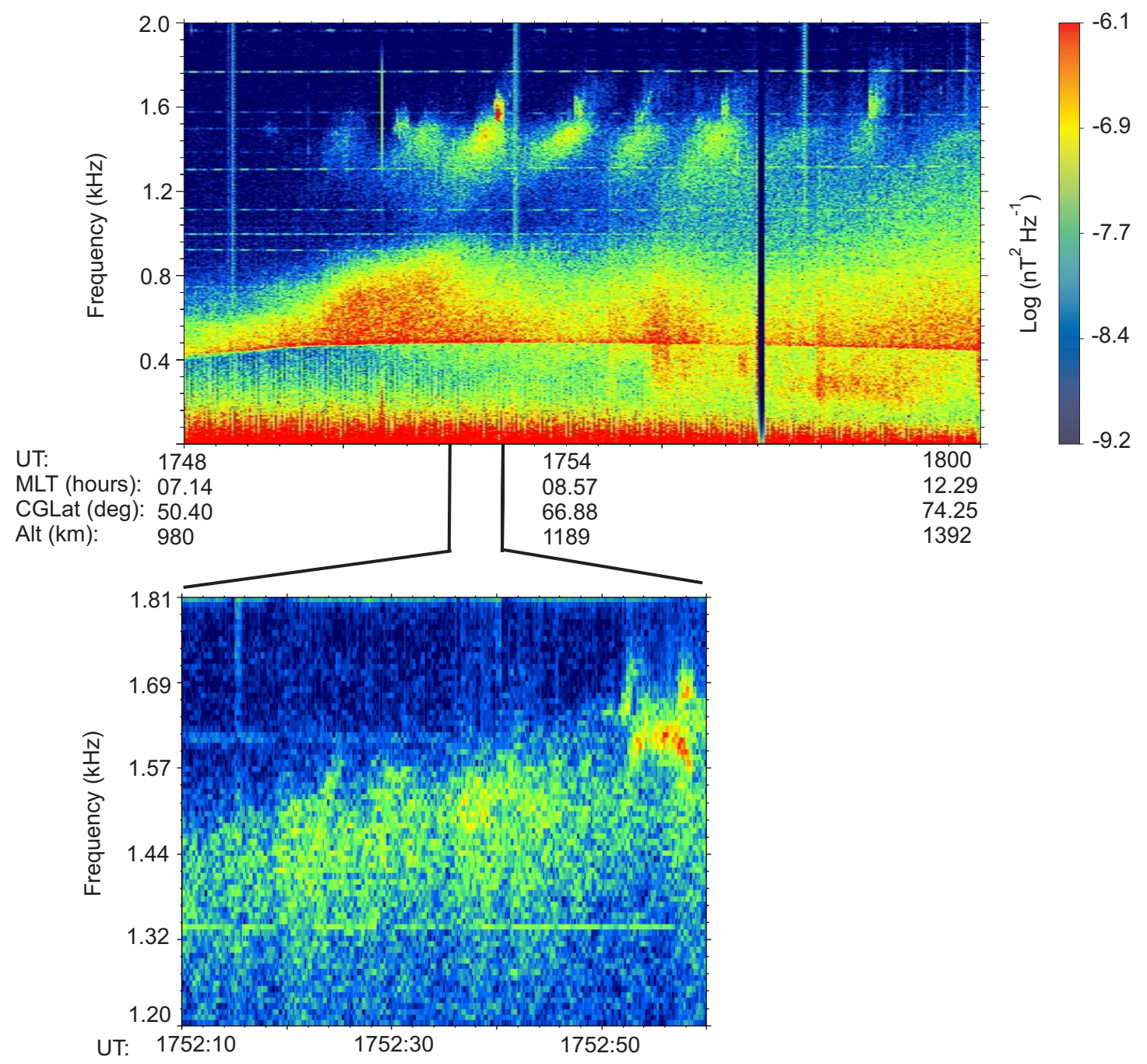

Fig. 3. Frequency-time spectra of the magnetic component of VLF emissions with simultaneous observation of QP hiss emissions and discrete VLF emissions on board Freja satellite on 3 April 1995. The whole event is shown on the plane (a) and detailed view of the single QP element is shown on plane (b).

\subsection{QP event observed by Magion 5}

We use VLF broad-band measurements carried out on board the Magion 5 satellite for analysis of QP emissions detected not too far from the magnetic equator, i.e. from the generation region. Magion 5 was launched as a part of the INTERBALL mission into a highly eliptic orbit with an apogee $20000 \mathrm{~km}$ and an inclination of $65^{\circ}$ (Triska et al., 1996). Magion 5 is constructed as a Sun-oriented and spin stabilized satellite with a spin period of about $2 \mathrm{~min}$.

The spacecraft was equipped with detectors of wave emissions, cold plasma density, and energetic particles. VLF broad-band measurements $(f<22.5 \mathrm{kHz})$ included both electric and magnetic field components. The measurements of basic parameters of cold plasma were carried out using the KM-14 detector designed to measure the ion density and electron temperature with a time resolution of $200 \mathrm{~ms}$. The ion density was measured within the range $N_{i}=10^{7}-5 \cdot 10^{12} \mathrm{~m}^{-3}$.
Energetic particles were detected using the DOK-S instrument. The DOK-S spectrometer measured energetic electrons with energies 60 to $800 \mathrm{keV}$ by means of two pairs of silicon detectors. The spin of Magion 5 allows us to estimate the angular distribution of energetic electrons averaged over 2 min.

QP emissions were observed on two orbits of the Magion 5 satellite, below we discuss one of these events, for which the cold plasma measurements are available, in addition to the wave data.

Event of 18 November 1998: Let us consider an example of QP emissions observed by Magion 5 in the orbit 3367 on 18 November 1998. During this event, Magion 5 moved in the morning sector from low $L$ shells into the auroral zone and detected QP emissions by both magnetic and electric antennas. 


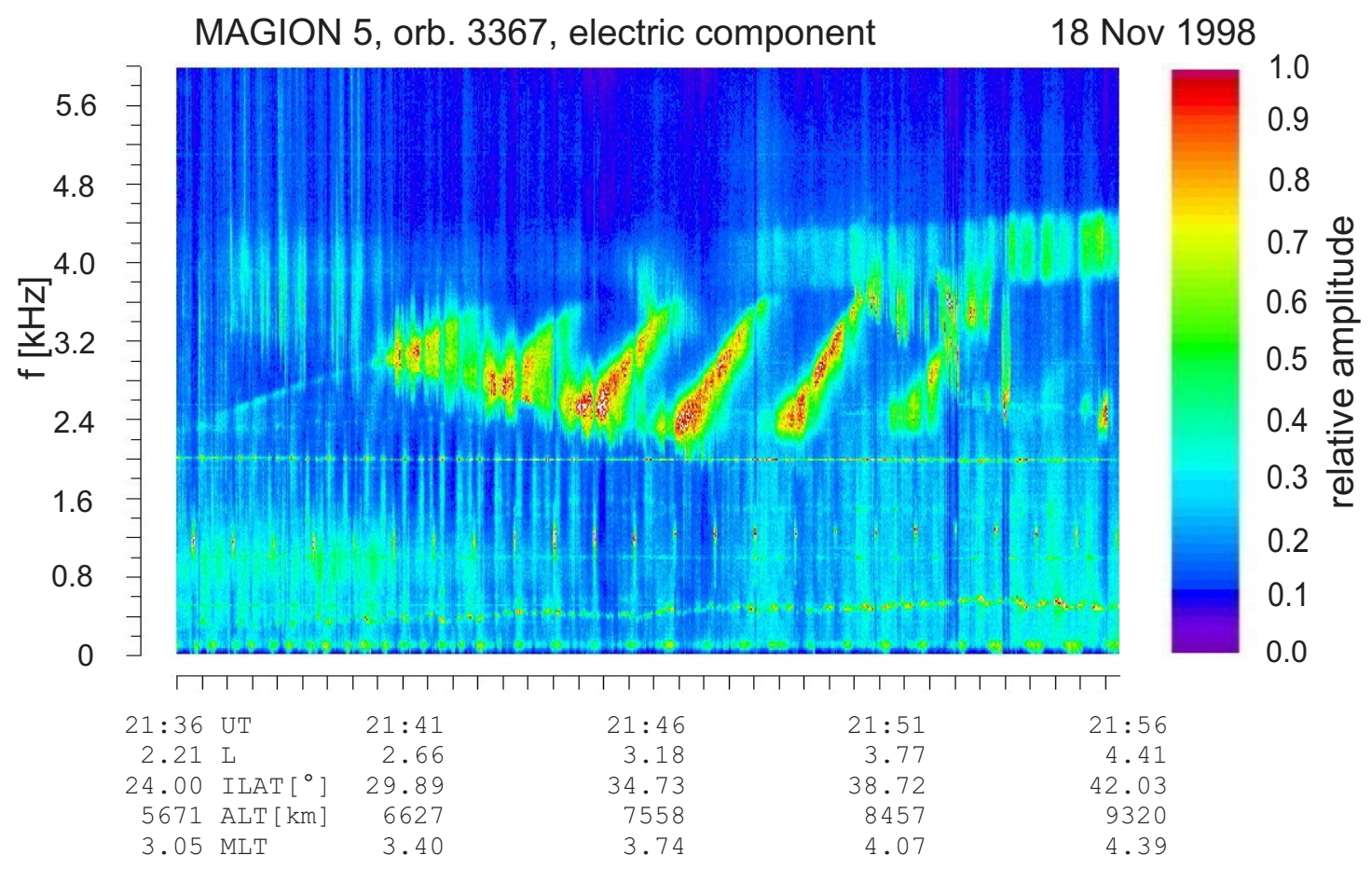

Fig. 4. Frequency-time spectra of the electric component of VLF emissions observed on board Magion 5 satellite on 18 November 1998.

The spectrogram of VLF emissions obtained by Magion 5 in the electric component is shown in Fig. 4. Clearly distinguished QP emissions were detected from 21:44 to 21:51 UT at $L$ shells from 3 to 4 in the early morning sector near 04:00 MLT. The spacecraft was located about $35^{\circ}$ from the equator. The geomagnetic disturbance was low $(\mathrm{Kp}=2-)$. The spectrum of QP emissions detected by Magion 5 is similar to those typically observed on the ground and having rising tones with diffuse structure (Sato and Fukunishi, 1981). QP emissions were detected at frequencies $1.9-3.6 \mathrm{kHz}$, their repetition period was about $2 \mathrm{~min}$, and all QP elements had practically the same constant slope of about $15 \mathrm{~Hz} / \mathrm{s}$.

The cold plasma number density was almost the same (about $80 \mathrm{~cm}^{-3}$ ) in the entire region where QP emissions were observed. This allows us to estimate the energy of resonant electrons generating these emissions as $W_{0}=150 \mathrm{keV}$ (see Eq. (8) below). The electron flux measured by the DOK-S spectrometer in this energy range (in the channel 90$200 \mathrm{keV}$ ) was $S \approx 4 \cdot 10^{5} \mathrm{~cm}^{-2} \mathrm{~s}^{-1}$. To estimate the flux in the equatorial plane, we should take into account that during the event considered, Magion 5 was at the geomagnetic latitude $38^{\circ}$, and the maximum equatorial pitch-angle corresponding to the Magion 5 location was $25^{\circ}$. The model AE-8 (Vette, 1991) gives a similar value of the electron flux in this pitch angle range. If we assume that the actual pitch-angle distribution corresponds roughly to the AE- 8 model, then we can estimate the flux at higher pitch angles. According to the AE-8 model, the electron flux at the equatorial pitch angle $\Theta_{L}=90^{\circ}$ increases by a factor of $4-5$ as compared to the flux at $\Theta_{L}=25^{\circ}$. Assuming such an angular distribution, we obtain the following estimate for the integral equatorial electron flux in the energy range 90-200 keV: $S \approx 2 \cdot 10^{6} \mathrm{~cm}^{-2} \mathrm{~s}^{-1}$. This value can be used as a lower limit for the integral equatorial flux of electrons with energies above $90 \mathrm{keV}$, since the particles with energies above $200 \mathrm{keV}$ were not taken into account.

Another interesting feature of this event is that the DOK$\mathrm{S}$ data in the channel $90-200 \mathrm{keV}$ indicate the existence of a butterfly-like pitch-angle distribution of electrons, i.e. nonmonotonic distribution when the number of particles at pitchangles close to $90^{\circ}$ is less than at some lower pitch-angles. This type of distribution was seen at time interval 21:3621:43 UT at L-shells below 3 and at ILAT below $30^{\circ}$. Later on, Magion 5 moved toward the higher L-shells and ILATs, and the butterfly-like pitch-angle distribution disappeared.

\section{Description of the model}

Since the emissions analyzed in this paper belong to the QP2 type, we assume that they are not related to the external modulation of the cyclotron instability (e.g. by a hydromagnetic waves, see Coronity and Kennel (1970)).

We use the model based on the self-consistent set of equations of the quasi-linear plasma theory for the distribution function $F(\mu, v, t)$ of energetic electrons and whistler wave spectral energy density $\varepsilon(\omega, t)$, averaged over oscillations of waves and particles between the mirror points and over the 
cross section of the interaction region (see Pasmanik et al. (2004) for more detail):

$$
\begin{aligned}
& \frac{\partial F}{\partial t}=\frac{1}{T}{ }_{b} \frac{\partial}{\partial \mu} \mu D \frac{\partial F}{\partial \mu}-\delta \cdot F+J \\
& \frac{\partial \varepsilon}{\partial t}=\frac{2}{T_{g}}(\Gamma-|\ln R|) \varepsilon,
\end{aligned}
$$

where $\mu=\sin ^{2} \Theta_{L}, \Theta_{L}$ is the equatorial pitch angle, $v$ is the electron velocity, $T_{b}$ is the bounce-oscillation period, $D$ is the coefficient of pitch angle diffusion, $J$ is the effective source of energetic electrons, $\delta$ is their loss rate due to precipitation, $\Gamma$ is the one-hop amplification of whistler waves on the pass between conjugate ionospheres, $T_{g}$ is the period of wave-packet oscillations, and $R$ is the effective reflection coefficient describing wave energy losses.

In a self-consistent model, the diffusion coefficient $D$ is determined by the wave intensity $\varepsilon$ and, in turn, the wave amplification $\Gamma$ is determined by the electron distribution function $F$ :

$D(\mu, v, t)=\int G_{1}(\omega, \mu, v) \varepsilon(\omega, t) d \omega$

$\Gamma(\omega, t)=\int G_{2}(\omega, \mu, v)\left(\mu \frac{\partial F}{\partial \mu}-\frac{\omega}{\omega_{B L}} F\right) d^{3} v$

where $G_{1}$ and $G_{2}$ are known functions (see Pasmanik et al., 2004), $\omega_{B L}$ is the electron gyrofrequency in the equatorial plane.

As the source of free energy, we consider the injection of energetic electrons with anisotropic velocity distribution into the interaction region in the process of their magnetic drift. Removal of energetic particles from the interaction region can be due to two mechanisms: (1) precipitation of energetic particles via the loss cone and (2) drift of particles away from the interaction region across the magnetic field lines (Trakhtengerts et al., 1986). Energetic particle losses due to their precipitation via the loss cone are taken into account by the term $-\delta \cdot F$ in Eq. (1). If the backscattering effect is neglected, the factor $\delta$ can be written as

$\delta=\left\{\begin{array}{ll}0 & \mu \geq \mu_{c} \\ \left(T_{b} / 4\right)^{-1} & 0 \leq \mu \leq \mu_{c}\end{array}\right.$,

where $\mu_{c}$ is the loss cone boundary. Inclusion of the backscattering would lead to a correction (decrease) for $\delta$. This correction is rather small in the regimes of weak and moderate pitch-angle diffusion, which are actually realised in the cases considered below. The second loss mechanism is taken into account in the term $J(\mu)$, which is determined by the difference of the pitch angle distributions of energetic particles drifting into and away from the interaction region.

To simplify the analysis of this model, we use the approximation of a monoenergetic electron distribution:

$\Phi(\mu, v, t)=\left(2 \pi v_{0}^{2}\right)^{-1} \delta\left(v-v_{0}\right) \tilde{\Phi}(\mu, t)$, where $W_{0}=m v_{0}^{2} / 2$ is the characteristic energy of energetic electrons, and consider only waves propagating along the geomagnetic field $(\boldsymbol{k} \| \boldsymbol{B})$. As was discussed by Bespalov and Trakhtengerts (1976), Demekhov and Trakhtengerts (1994) and Pasmanik et al. (2004), the monoenergetic approximation does not lead to significant changes in the wave spectrum dynamics for ducted whistler waves, since the inhomogeneity of the geomagnetic field provides a spread in resonant energies, and the growth rate of ducted whistler waves is determined by the integral parameters of the energy distribution, such as the characteristic energy and the total electron flux. Numerical estimations show that the whistler wave amplification $\Gamma$ in the inhomogeneous geomagnetic field for a monoenergetic distribution is almost equal to the case of the finite velocity spread $\Delta V / V_{0}=0.1$ (for the parameter values used below). For $\Delta V / V_{0}=0.2$ the maximum amplification decreases by a factor 2 , and the generation band is broadened by $15 \%$. In the case of an even wider spread, the qualitative profile of amplification remains the same with the amplification band broadened (up to 50\%) and the maximum amplification decreased. Thus, generation of somewhat broader emission spectrum than in our model simulations should be expected in the case of a smooth energy distribution.

\section{Numerical results}

Detailed analysis of this model using various combinations of parameters and possible generation regimes was performed by Pasmanik et al. (2004). Here, we briefly summarize relevant results for convenience of the reader.

In the case where losses due to precipitation are dominant, generation of a relatively wide intensity spikes with rather small modulation of the wave intensity takes place. In the case where the drift losses are dominant, narrower spikes are more common, and the generation regime depends strongly on the source pitch-angle distribution. In the case of a monotonic pitch-angle distribution and rather low source intensity, a periodic generation occurs. For higher source intensities the alteration of spikes with different frequency drift rate can take place. A mild butterfly feature at the pitch-angle distribution in the source (i.e. with not very pronounced maximum) gives almost no change in the generation regime. A butterfly distribution with extremely high maximum leads to a strong wave damping at higher frequencies resonant with pitch-angles corresponding to the negative derivative $\partial \Phi / \partial \mu<0$, so the generation is possible only in the low-frequency band. In the case of a moderate "butterflylike" maximum, a persistent low frequency band with periodic modulation of its intensity and periodic spikes at higher frequencies can take place. Since the negative derivative of the distribution function can be accounted as an effective increase of the wave damping, the intensity of the wave spikes decreases as compared with the case of a monotonic distribution.

Below we present some specific modeling results to demonstrate that the FCM model can be used for explanation 
of the specific features of QP emissions presented in Sect. 2. On the basis of this model, we can explain not only temporal characteristics of QP emissions (such as spike duration and repetition period), but also the spectral characteristics, such as the frequency band and peculiarities of spectral shapes of QP emission elements.

To model a particular event, one needs to know the system parameters. Unfortunately, the data available from the observations are not complete, i.e. they do not allow us to determine all model parameters unambiguously. Thus, it is necessary to make some assumptions based on the available data and the general results of model analysis.

As it follows from the results of Pasmanik et al. (2004), variation in some model parameters lead only to minor quantitative changes in the properties of the generation regime (such as the wave generation band, the period of generation, the spike duration, etc.). But there are several parameters which determine the generation regime qualitatively. Such key parameters are the mechanism of removal of the energetic particles from the interaction region, the pitch-angle distribution in the source, and the source intensity. Due to the lack of experimental data, the following two-step method was used to obtain the model parameters. At first, the key parameters for each event were estimated by qualitative comparison of the general characteristics and peculiarities of observed wave spectrum with the results of Pasmanik et al. (2004). After that, adjustment of other model parameters was made to obtain the best coincidence of the observed and simulated generation regimes.

Some specific details of estimation of the model parameters are discussed below.

Since we consider only ducted whistler waves, we can assume that the QP emissions for all events were generated at approximately the same magnetic shell $L$ as they were detected. Since the satellite $L$-shell was changing during an event, an average value was chosen.

The lowest frequency of the generation band, determined by the cyclotron resonance condition in the equatorial plane, is approximately equal to

$\omega_{\min } \simeq \omega_{0}=\omega_{B L} / \beta_{*}$,

where

$\beta_{*}=\left(\frac{\omega_{p L} v_{0}}{\omega_{B L} c}\right)^{2}=N_{c} W_{0}\left(\frac{8 \pi e^{2} m}{c^{2} \omega_{B L}^{2}}\right)$,

$\omega_{p L}=4 \pi e^{2} N_{c} / m$ is plasma frequency in the equatorial plane, and $N_{c}$ is the cold plasma density. Thus, one can estimate the value of $N_{c} W_{0}$ from the observed wave spectrum if the $L$-shell is known. Note that the model output is much more sensitive to the product $N_{c} W_{0}$ than to $N_{c}$ or $W_{0}$ alone. Therefore, it is impossible to resolve this ambiguity by modeling only.

The frequency dependence of wave losses $|\ln R(\omega)|$ can be estimated from the upper frequency of the generation band, which is mainly determined by this parameter (i.e. increase of $|\ln R|$ with an increase in $\omega$ leads to a decrease in the upper frequency).
Other model parameters, such as the pitch angle distribution and intensity of the source, wave loss rate, and the interaction region size (in the case of the drift removal), are rather difficult to retrieve only from comparison of the model results with experiment in the absence of the direct measurements for at least some of these parameters. Nevertheless, we show that modeling a particular event can be used in comparison with available data to estimate the missing parameters or at least to obtain the range of their values, in which the generation properties remain almost the same.

\subsection{Modeling QP events observed by Freja}

Event of 18 June 1993: As it was mentioned above, the specific feature of the dynamic spectrum in this event (Fig. 1) is an increase in the frequency drift rate during the generation of a single QP element. According to Pasmanik et al. (2004), such a spectrum is typical when an interaction region is rather small (duct), i.e. the energetic particles removal is mainly due to their drift, and if a source with rather high pitch angle anisotropy is present.

According to the procedure described above, the following model parameters were chosen. The average $L$-shell value of this event was $L=3.8$. The cold plasma density was evaluated from the simplest expression $N_{c} \approx 100(4.4 / L)^{4.4}$, which gives not too large an error as compared with more complex models, see, for example, Gallagher et al. (2000). For the selected $L$-shell this simple formula yields $N_{c} \approx 170 \mathrm{~cm}^{-3}$. The corresponding characteristic energy of electrons, estimated from Eq. (7), is $W_{0}=40 \mathrm{keV}$ ( $f_{\min } \sim 2 \mathrm{kHz}$, see Fig. 1). To obtain the described spectrum peculiarity (see above and Pasmanik et al. (2004)), we took into account only the drift removal mechanism (i.e. $\delta \equiv 0$ ) and chose a monotonic pitch angle distribution of energetic electrons in the source (i.e. the initial pitch angle distribution of drifting electrons):

$F_{\text {in }}=\left\{\begin{array}{ll}C \sin ^{\alpha}\left(\frac{\pi}{2} \mathfrak{x} / \mathfrak{x}_{0}\right), & \mathfrak{x} \leq \mathfrak{x}_{0} \\ C, & \mathfrak{x}>\mathfrak{x}_{0}\end{array}\right.$,

where $æ \equiv \sqrt{\mu}, C$ is the normalizing constant (Pasmanik et al., 2004). To obtain the generation period of $200 \mathrm{~s}$ and an upper frequency of $\sim 5 \mathrm{kHz}$, the following choice of other model parameters was made: the duct diameter in the equatorial plane $120 \mathrm{~km}$, the energetic electron flux $S \approx 6 \cdot 10^{7} \mathrm{~cm}^{-2} \mathrm{~s}^{-1}$, wave losses are $\left|\ln R=2\left(\omega / \omega_{0}\right)^{2}\right|$, and the source parameters are $\mathfrak{x}_{0}=0.1$ and $\alpha=1$. As it was mentioned above, on the basis of the available experimental data it is impossible to determine the parameters unambiguously. In particular, the model gives a similar output for $N_{c} \approx 120 \mathrm{~cm}^{-3}$ (a value following from Gallagher et al. (2000)) and $W_{0} \approx 55 \mathrm{keV}$. The period of pulsations is roughly proportional to the ratio of the wave losses $|\ln R|$ to the source intensity (characterized by the flux value $S$ ). Thus, the same generation regime will occur if the wave losses and the source intensity are changed by the same factor. As well, the increase in $\alpha$ or $æ_{0}$ can be compensated by a corresponding increase in the source intensity and $N_{c} W_{0}$ factor. The similar model output occurs if we use a non-monotonic 


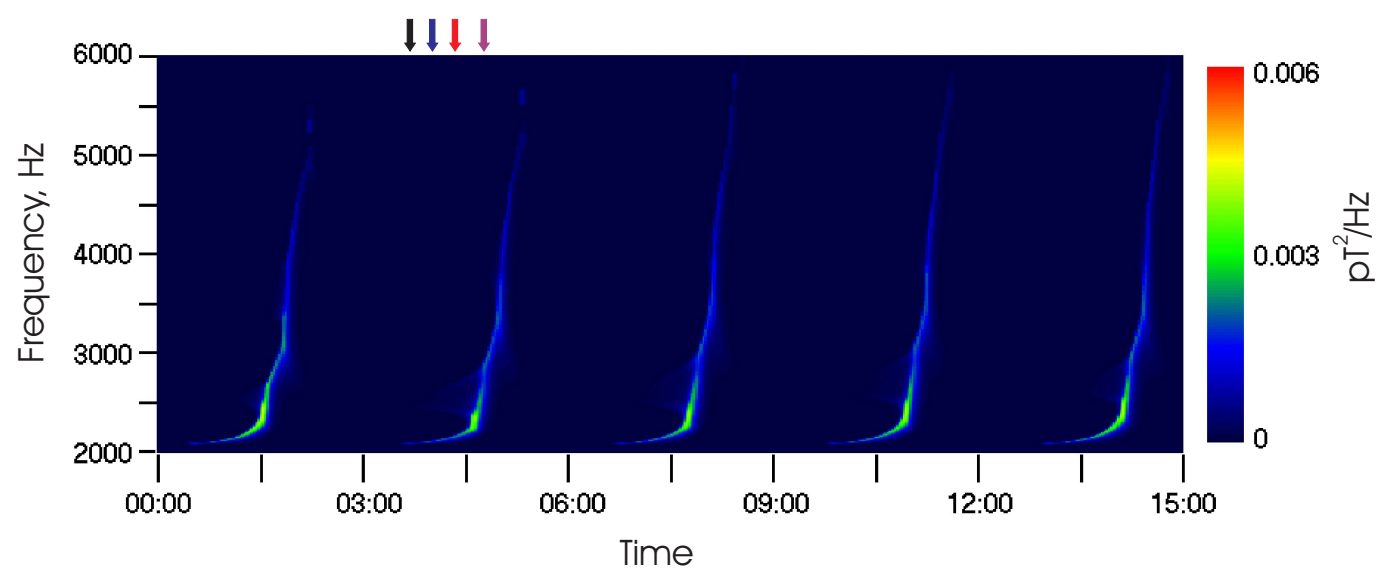

Fig. 5. Spectrogram of the wave intensity obtained from the FCM model for the experimental case presented in Fig. 1.

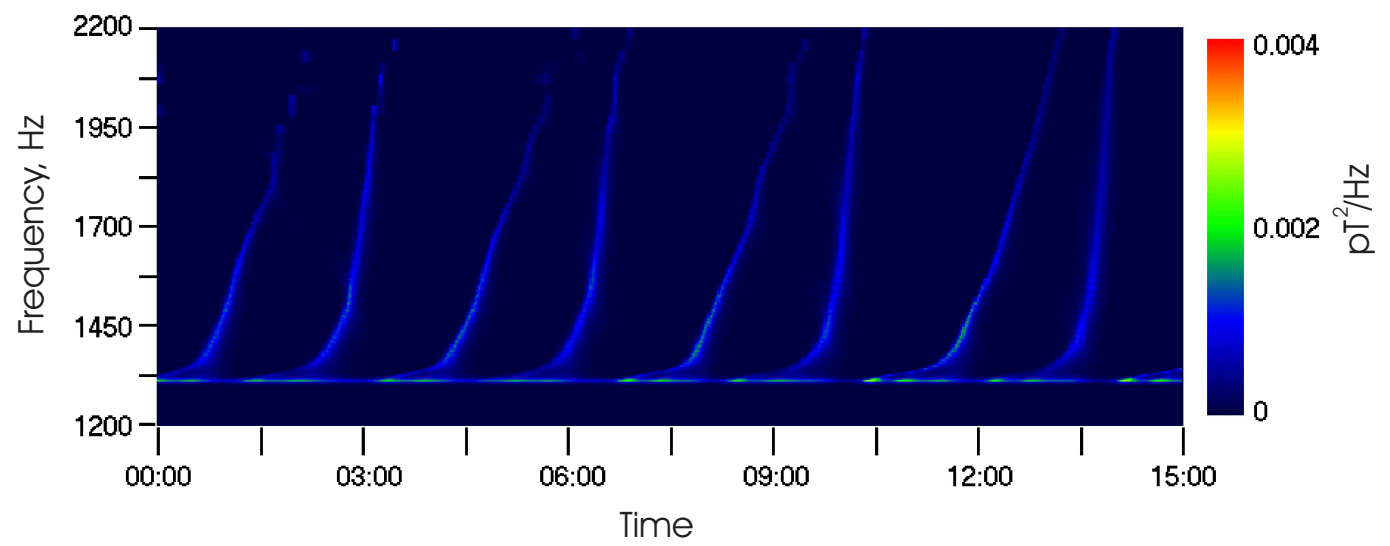

Fig. 6. Spectrogram of the wave intensity obtained from the FCM model for the experimental case presented in Fig. 2.

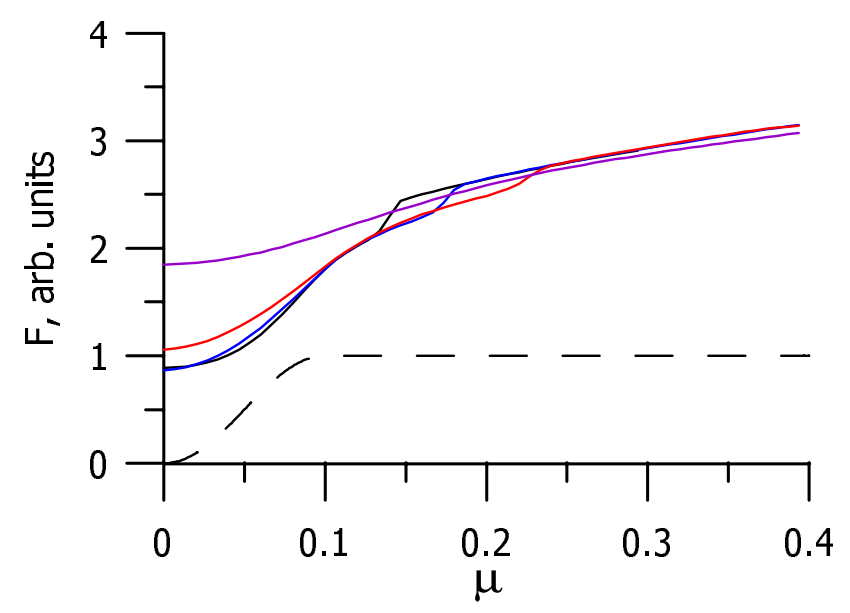

Fig. 7. Formation of a sharp gradient on the pitch angle distribution of energetic electrons during generation of an element of QP emission in the case from Fig. 5. Time marks for snapshots are given in the corresponding color at the top of the spectrogram in Fig 5. Pitch angle distribution in the source is shown by the dashed line. (butterfly-like) source pitch-angle distribution with weakly pronounced maximum (small value of $\Delta$, see Eq. (10) below), but the more simple shape of the source distribution was used to model this event.

The result of numerical simulation for this event is shown in Fig. 5. As one can see, the generation of a wave intensity spike is accompanied by a shift of the maximum of wave amplitude to higher frequencies, with the shift rate increasing during generation. Such a regime occurs due to formation of a rather sharp gradient at the energetic electron pitch angle distribution at the boundary between resonant and non-resonant particles, which moves toward higher pitch angles during the cyclotron instability development (Demekhov and Trakhtengerts, 1994).

Event of 12 November 1993: Peculiarity of the second event from Freja satellite (Fig. 2) is the alteration of QP elements with different frequency drift rates. According to Pasmanik et al. (2004), such a regime typically occurs if there are the same characteristics of the removal mechanism and the pitch-angle distribution in the source as for the previous event, but the source intensity is higher. 


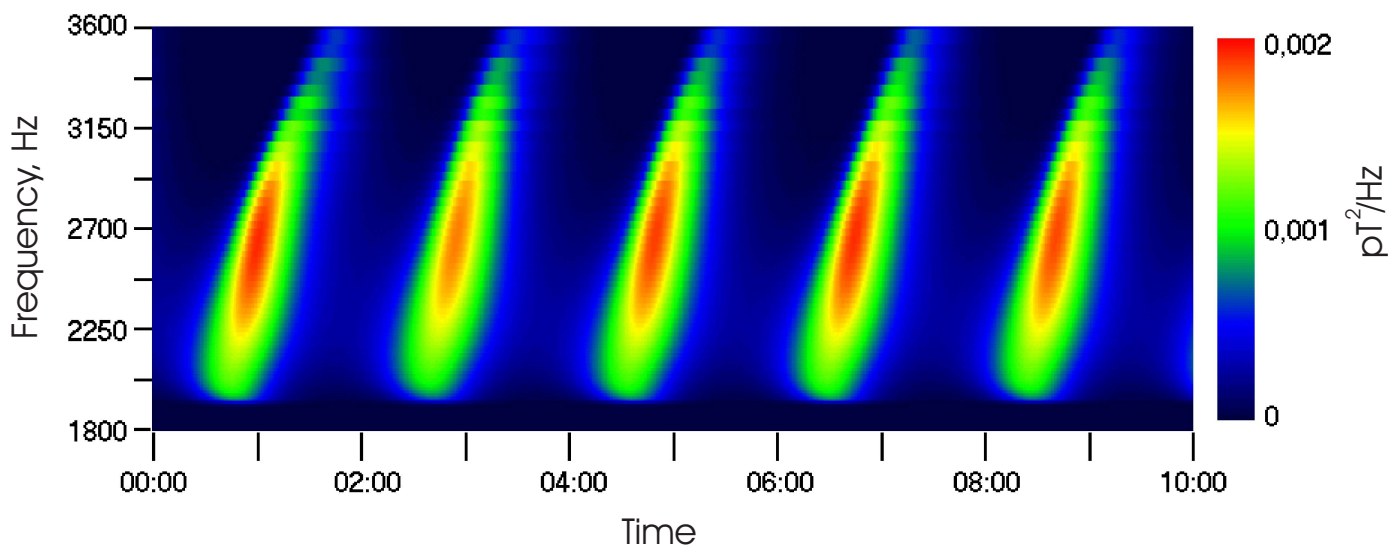

Fig. 8. Spectrogram of the wave intensity obtained from the FCM model for the experimental case presented in Fig. 4.

The results of the numerical simulation for this event is shown in Fig. 6. The average $L$-shell value for this event was $L=4$. Using the same approximate expression for the equatorial cold plasma density distribution as above we obtain $N_{c}=130 \mathrm{~cm}^{-3}$ and the corresponding energy of electrons estimated from Eq. (7) is $W_{0}=45 \mathrm{keV}\left(f_{\min } \sim 1.4 \mathrm{kHz}\right.$, see Fig. 2). As in the previous case, only drift removal is taken into account and the same pitch angle distribution is used. Also, the same size of the interaction region and wave losses were used. For these parameters the generation period of $100 \mathrm{~s}$ was obtained for the energetic-electron flux $S \approx 2 \cdot 10^{8} \mathrm{~cm}^{-2} \mathrm{~s}^{-1}$.

As is seen from Fig. 6, the alteration of QP elements with different frequency drift rates was obtained, which is similar to that observed on board Freja satellite.

The occurrence of such a generation regime is explained as follows (see also Pasmanik et al. (2004)). When the intensity of the source is rather small, its contribution during the generation of a single spike is negligible. Therefore, we may consider that the generation of each spike starts from the same conditions in the generation region (see, e.g. Fig. 5). With an increase in the source intensity, the modification of the energetic electron distribution during a spike under the action of the source becomes more significant. This leads to an increase in the distribution anisotropy at low pitch angles, but the pitch angle diffusion due to the waves generated by a sharp gradient at higher $\mu$ values does not allow the instability threshold to be reached. Thus, during the generation of the first spike, the source sustains the system near the threshold, so after the end of the spike the system quickly reaches this threshold, and the second spike with a higher frequency drift rate is generated. After that, the system comes to the initial state below the threshold.

As it was mentioned above, the generation of a single element of QP emission is related to the formation of a sharp gradient in the pitch angle distribution of energetic electrons and its motion towards higher pitch angles. This is illustrated in Fig. 7, where the snapshots of the pitch angle distribution of energetic electrons at different generation stages of a QP element are shown. The existence of this dynamical feature is very important for the explanation of the relation between QP and discrete VLF emissions. According to recent theoretical studies, such a step-like deformation can be a source of discrete ELF/VLF emissions (Trakhtengerts, 1995, 1999; Demekhov et al., 2003).

\subsection{Modeling the QP event observed by Magion 5}

As it was mentioned in Sect. 2, the data from Magion 5 satellite include cold plasma measurements. According to these measurements, the cold plasma density was almost constant for the entire event presented in Fig. 4. Thus, we should assume that the interaction region in this case is rather large, and the dominating mechanism of energetic particle removal is due to their precipitation.

The simulation results for this event are presented in Fig. 8. The average $L$-shell value of this event was $L=3.6$. The measured cold plasma density was $N_{c}=80 \mathrm{~cm}^{-3}$, and the corresponding characteristic energy of electrons estimated from Eq. (7) is $W_{0}=150 \mathrm{keV}\left(f_{\min } \sim 1.8 \mathrm{kHz}\right.$, see Fig. 4).

The observed upper limit of the generation band can be obtained in simulations for these parameter values in two cases: (a) a strong increase in whistler wave losses at $f \geq 3.6 \mathrm{kHz}$ (corresponding to $\omega / \omega_{H L} \sim 0.2$ for $L=3.6$ ) and monotonic source like Eq. (9), or (b) a butterfly-like distribution in the source, since the negative derivative $\partial \Phi / \partial \mu<0$ leads to an increase in the wave damping in the corresponding frequency range (see Pasmanik et al., 2004, for a more detailed discussion). The second hypothesis appears to be more consistent with the observations, which indicate the butterfly-like pitchangle distribution of energetic electrons. Thus, to model this case we used a smooth frequency dependence of the wave damping $|\ln R|=3\left(\omega / \omega_{0}\right)^{-1}$ and the butterfly-like pitch angle distribution in the source:

$$
F_{\text {in }}=\left\{\begin{array}{lr}
C \cdot[1+\Delta] \cdot \sin ^{\alpha}\left(\frac{\pi}{2} \mathfrak{x} / \mathfrak{x}_{0}\right), & \mathfrak{x} \leq \mathfrak{x}_{0} \\
\left.C \cdot\left[1+\Delta-\Delta \cdot \sin ^{\alpha_{1}}\left(\frac{\pi}{2}\left(\mathfrak{x}-\mathfrak{x}_{0}\right) / \mathfrak{x}_{1}\right)\right)\right], & \mathfrak{x}_{0}<\mathfrak{x} \leq \mathfrak{x}_{1}+\mathfrak{x}_{0} \\
C, & \mathfrak{x}>\mathfrak{x}_{1}+\mathfrak{x}_{0}
\end{array} .\right.
$$


Here, the parameter $\Delta$ characterizes the height of the maximum, while the parameters $æ_{1}$ and $\alpha_{1}$ characterize the width and steepness of its negative slope at $\mathfrak{x}>\mathfrak{x}_{0}$ (Pasmanik et al., 2004).

The observed generation band and the generation period about $120 \mathrm{~s}$ were obtained for the following source parameters: $\mathfrak{x}_{0}=0.5, \alpha=3, \mathfrak{x}_{1}=0.4, \alpha_{1}=1, \Delta=0.5$, and $S \approx 4 \cdot 10^{6} \mathrm{~cm}^{-2} \mathrm{~s}^{-1}$. The choice of $\alpha_{1}, \mathfrak{x}_{1}$ and $\Delta$ parameters is made to match the observed upper generation frequency. As it was mentioned above, small $\Delta$ values do not give significant changes to the generation regime. An increase in $\Delta$ leads to a decrease in the upper frequency, and for extremely high values of $\Delta$ the generation occurs only at frequencies $\omega<\omega_{B L} / \beta_{*} /\left(1-\mathfrak{x}_{0}\right)$. Similarly to that, an increase in $\alpha_{1}$ or decrease in $\mathfrak{x}_{1}$ leads to a decrease in the upper frequency.

\section{Conclusions}

In this paper we present the results of both experimental and theoretical analysis of QP2 events detected by the Freja and Magion 5 satellites. Detailed analysis of available QP events revealed certain specific features of their dynamic spectra, which have not been reported earlier. These are (1) an increase in the frequency drift rate during the generation of a single element of QP emission, (2) the alteration of QP elements with different frequency drift rates, (3) the relationship between QP hiss emissions and discrete VLF emissions observed simultaneously.

A numerical model based of the self-consistent equations of quasi-linear plasma theory was employed to explain the formation of these emissions. We show that this model is able to explain these new and interesting features of the dynamic spectra of the selected QP emissions. Moreover, detailed knowledge of the relation between the model parameters and output enables us to obtain significant restrictions on the possible values of the source parameters for the discussed QP events.

It would be very interesting to apply this model for simulating other events with QP emissions observed by satellites, for which the simultaneous measurements of the wave field and plasma parameters are available.

It would be especially interesting to compare the cases of QP2 and QP1 events (see, e.g. Morrison and Freeman, 1995; Sazhin and Hayakawa, 1994; Smith et al., 1998, for detailed experimental analysis of the latter phenomena). The presented model can be easily supplied by the term describing the flux-tube modulation by the Pc 3-4 hydromagnetic pulsations (Bespalov and Trakhtengerts, 1978; Bösinger et al., 1996) and such a study is planned for the future.

Acknowledgements. The work of DLP, AGD, VYT and EET was supported by the Russian Foundation for Basic Research (grants No. 02-02-17109 and 04-05-64955) and by the Program For Basic Research of the Russian Academy of Science. EET and VYT acknowledge partial support from the International Space Science Institute. OS acknowledges support from grants MSM 113200004 and GACR 202/03/0832. FJ acknowledges support from grant
A3042201 of GA CA. KK acknowledges Slovak VEGA grant agency project 4064 for support. Part of this work was done during the visit of DLP, EET, AGD, VYT and OS to the Sodankylä Geophysical Observatory, funded by the EU LAPBIAT program. Data processing of DOK-S measurements is done by J. Stetiarova, pitch angle computation by D. Ciobanu, Bucharest, Romania. We thank B. Holback and IRF-U for the permission to use Freja data. We acknowledge SPENVIS system and D. Heynderickx for providing data on AE-8 model (www.spenvis.oma.be).

Topical Editor T. Pulkkinen thanks two referees for their help in evaluating this paper.

\section{References}

Bespalov, P. A. and Trakhtengerts, V. Y.: The dynamics of the cyclotron instability in a magnetic trap, Fizika Plazmy, 2, 397-406, 1976.

Bespalov, P. A. and Trakhtengerts, V. Y.: Self-modulation of the cyclotron instability by Alfvén waves, Fizika Plazmy, 4, 177183, 1978.

Bespalov, P. A.: Self-modulation of the plasma cyclotron maser emission, Pis'ma v ZhETF, 33, 192-195, 1981.

Bösinger, T., Kaila, K., Rasinkangas, R., Pollari, P., Kangas, J., Trakhtengerts, V. Y., Demekhov, A. G., and Turunen, T.: An EISCAT study of a pulsating auroral arc: (I) simultaneous ionospheric electron density, auroral luminosity and magnetic field pulsations, J. Atmos. Terr. Phys., 58, 23-35, 1996.

Coronity, F. V. and Kennel, C. F.: Electron precipitation pulsations, J. Geophys. Res., 75, 1279-1289, 1970.

Davidson, G. T.: Self-modulated VLF wave-electron interactions in the magnetosphere: A cause of auroral pulsations, J. Geophys. Res., 84, 6517-6523, 1979.

Davidson, G. T.: Pitch angle diffusion in morningside aurorae 2. The formation of repetitive auroral pulsations, J. Geophys. Res., 91, 4429-4436, 1986.

Demekhov, A. G. and Trakhtengerts, V. Y.: A mechanism of formation of pulsating aurorae, J. Geophys. Res., 99, 5831-5841, 1994.

Demekhov, A. G., Nunn, D. and Trakhtengerts, V. Y.: Backward wave oscillator regime of the whistler cyclotron instability in an inhomogeneous magnetic field, Phys. Plasmas, Vol. 10, No.11, 4472, 2003.

Gallagher, D. L., Craven, P. D., and Comfort, R. H.: Global core plasma model, J. Geophys. Res., 105, 18 819-18 834, 2000.

Hayakawa, M. and Sazhin, S. S.: Mid-latitude and plasmaspheric hiss: A review, Planet. Space Sci., 40, 1325-1338, 1992.

Helliwell, R. A.: Whistlers and Related Ionospheric Phenomena, Stanford Univ. Press, Palo Alto, Calif., 1965.

Holback, B., Jansson, S. E., Ahlin, L., Lundgren, G., Lyngdal, L., Powell, S., and Meyer, A.: The Freja wave and plasma-density experiment, Space Sci. Rev., 70, 577-592, 1994.

Hovner, M. S., Kuznetsova, V. A., and Likhter, I. I.: On the midlatitude modulation of fluxes of energetic electrons and VLF emissions, Geomagn. and Aeron. (English Transl), 17, 867-870, 1977.

Jiricek, F., Triska, P., Jurov, V. E., and Titova, E. E.: On the characteristics of VLF emissions in the upper ionosphere and on the ground, Studia Geophysica et Geodaetica, 25, 81-86, 1981.

Morrison, K. and Freeman, M. P.: The role of upstream ULF waves in the generation of quasi-periodic ELF-VLF emissions, Ann. 
Geophysicae, 13, 1127-1133, 1995.

Pasmanik, D. L., Demekhov, A. G., Trakhtengerts, V. Y., and Parrot, M.: Modeling whistler wave generation regimes in magnetospheric cyclotron maser, Ann. Geophys., 22, 3561-3570, 2004.

Sato, N. and Fukunishi, H.: Interaction between ELF-VLF emissions and magnetic pulsations: classificaton of quasi-periodic ELF-VLF emissions based on frequency-time spectra, J. Geophys. Res., 86, 19-29, 1981.

Sato, N., Hayashi, K., Kokubun, S., Oguti, T., and Fukunishi, H.: Relationship between quasi-periodiv VLF-emission and geomagnetic pulsation, J. Atmos. Terr. Phys., 36, 1515-1526, 1974.

Sazhin, S. S. and Hayakawa, M.: Periodic and quasiperiodic VLF emissions, J. Atmos. Terr. Phys., 56, 735-753, 1994.

Smith, A. J., Engebretson, M. J., Klatt, E. M., Inan, U. S., Arnoldy, R. L., and Fukunishi, H.: Periodic and quasiperiodic ELF/VLF emissions observed by an array of Antarctic stations, J. Geophys. Res., 103, 23 611-23 622, 1998.
Tixier, M. and Cornilleau-Wehrlin, N.: How are the VLF quasiperiodic emissions controlled by harmonics of field line oscillations? - The results of a comparison between ground and GEOS satellites measurements, J. Geophys. Res., 91, 6899-6919, 1986.

Trakhtengerts, V. Y., Tagirov, V. R., and Chernous, S. A.: Flow cyclotron maser and impulsive VLF emissions, Geomagn. Aeron. 26, 99-106, 1986.

Trakhtengerts, V. Y.: Magnetosphere cyclotron maser: Backward wave oscillator generation regime, J. Geophys. Res., 100, 17,205-17,210, 1995.

Trakhtengerts, V. Y.: A generation mechanism for chorus emission, Ann. Geophysicae, 17, 95-100, 1999.

Triska, P., Vojta, J., Base, J., Chum, J., Czapek, A., Hruska, F., Korab, J., Agafonov, Y., Khrapchenkov, V., Friedrich, M., and F., P.: Small satellites for the INTERBALL mission, in small satellites for earth observation, in Proc. of the International Symposium of the IAA, edited by Roeser, R. H. P. and Valenzuela, A., 212-215, 1996.

Vette, J. I.: The AE- 8 trapped electron model environment, NASA STI/Recon Technical Report N, 92, 24 228, 1991. 